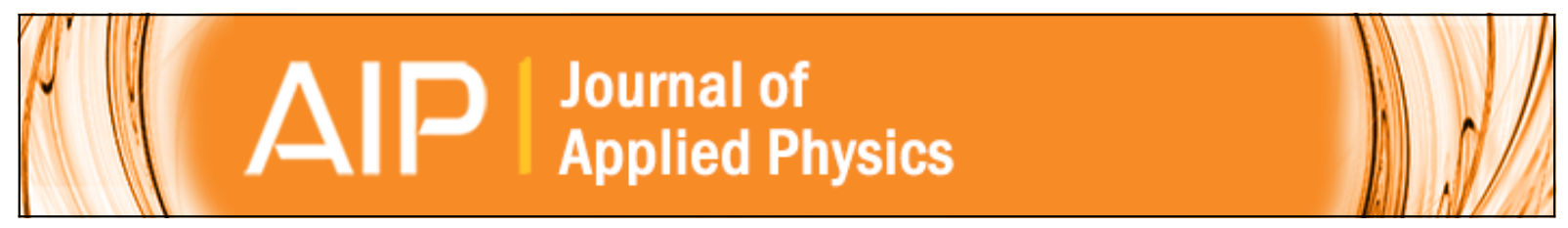

\title{
Electrical resistivity of acceptor carbon in GaAs
}

A. Ferreira da Silva, I. Pepe, Bo E. Sernelius, C. Persson, R. Ahuja, J. P. de Souza, Yoko Suzuki, and Y. Yang

Citation: Journal of Applied Physics 95, 2532 (2004); doi: 10.1063/1.1645971

View online: http://dx.doi.org/10.1063/1.1645971

View Table of Contents: http://scitation.aip.org/content/aip/journal/jap/95/5?ver=pdfcov

Published by the AIP Publishing

\section{AlP Re-register for Table of Content Alerts}

\section{Create a profile. \\ Sign up today!}




\title{
Electrical resistivity of acceptor carbon in GaAs
}

\author{
A. Ferreira da Silva ${ }^{a)}$ and I. Pepe \\ Instituto de Física, Laboratório de Propriedades Óticas, Universidade Federal da Bahia, \\ Campus Universitário de Ondina, 40210-340 Salvador, Bahia, Brazil \\ Bo E. Sernelius \\ Department of Physics and Measurement Technology, Linköping University, SE-58183 Linköping, Sweden \\ C. Persson ${ }^{\text {b) }}$ and R. Ahuja \\ Condensed Matter Theory Group, Department of Physics, Uppsala University, SE-75121 Uppsala, Sweden
}

J. P. de Souzac)

Instituto de Física, Universidade Federal do Rio Grande do Sul, 91501970 Porto Alegre, RS, Brazil

Yoko Suzuki and Y. Yang

Physics Department, City College of the City University of New York, New York 10031

(Received 23 September 2003; accepted 12 December 2003)

The electrical resistivity was investigated from room temperature down to $1.7 \mathrm{~K}$ for the shallow acceptor carbon in GaAs prepared by ion implantation with impurity concentrations between $10^{17}$ and $10^{19} \mathrm{~cm}^{-3}$. Good agreement was obtained between the measured resistivities and resistivities calculated by a generalized Drude approach at similar temperatures and doping concentrations. The critical impurity concentration for the metal-nonmetal transition was found to be about $10^{18} \mathrm{~cm}^{-3}$.

(C) 2004 American Institute of Physics. [DOI: 10.1063/1.1645971]

\section{INTRODUCTION}

GaAs-based semiconductors are well recognized as active materials in the design of bandgap-engineered devices and as constituents of lattice-matched heterostructures. ${ }^{1-3}$ The role of impurities in these materials is very important in the fabrication of different types of devices. For instance, acceptor carbon in GaAs is used for the high-concentration doping of a transistor base in the epitaxy of heterobipolar structures and for the formation of buried $p$-type layers in field-effect transistors. ${ }^{4,5}$

In this work, we have investigated the resistivity of acceptor carbon-doped GaAs (GaAs:C) in the temperature range from 1.7 to $300 \mathrm{~K}$ for impurity concentration varying from the insulating to the metallic range; that is from about $10^{17}$ to $10^{19} \mathrm{~cm}^{-3}$. The energy of activation to the conduction band is obtained from the resistivity at low impurity concentration. The samples were prepared by ion implantation in Van der Pauw structures delineated in GaAs chips. ${ }^{6,7}$ The resistivities obtained experimentally were compared with resistivity values calculated from a generalized Drude approach (GDA) $)^{8-11}$ at similar temperatures and dopant concentrations. The critical impurity concentration $N_{c}$ for the metal-nonmetal (MNM) transition was estimated from these results, and calculated using three different computational methods. ${ }^{12}$

\footnotetext{
${ }^{a)}$ Electronic mail: ferreira@ fis.ufba.br

${ }^{b}$ Present address: National Renewable Energy Laboratory, Golden, Colorado 80401 .

${ }^{c}$ Present address: Thomas J. Watson Research Center, IBM Yorktown Heights, NY, NY 10598.
}

\section{EXPERIMENTAL DETAILS}

The samples used in this work were semi-insulating liquid-encapsulated Czochralski-grown GaAs wafers of (100) orientation. After organic cleaning, the samples were ion implanted with $\mathrm{C}^{+}$. Implantation was performed at a low temperature of $100 \mathrm{~K}$ in order to obtain high electrical activation yields of implanted carbon. ${ }^{6}$ The doses and energies to create a flat doping profile were determined using TRIM code simulation. ${ }^{13}$ In order to minimize channeling effects, the samples were tilted $10^{\circ}$ and rotated $25^{\circ}$ with respect to the $\langle 110\rangle$ direction. The as-implanted samples were submitted to capless rapid thermal annealing (Si proximity) at $900^{\circ} \mathrm{C}$ for $10 \mathrm{~s}$ in an Ar atmosphere and then sintered at $\cong 300^{\circ} \mathrm{C}$ for 3 min. An ac bridge was used to measure the resistance of the devices in a He-4 cryostat, in which sample temperature was controlled to within $0.5 \%$ between 1.7 and $300 \mathrm{~K}$. The resistivity was determined by the van der Pauw method. ${ }^{7,10}$ Difficulties were encountered with the measurements at low temperatures for the samples with low dopant concentrations because of extremely high contact resistance; data were not obtained at the lowest temperatures for the three lowest concentrations (see Fig. 1).

\section{THEORY}

One starts from a generalized Drude expression for dynamical conductivity $\sigma(\omega)^{8,9}$

$$
\sigma(\omega)=\frac{N e^{2}}{m^{*}} \frac{1}{1 / \tau(\omega)-i \omega},
$$

with a generalized complex-valued and frequency-dependent relaxation time $\tau(\omega)$. In the GDA, the semiclassical conductivity expression in Eq. (1) is expanded for high frequencies 


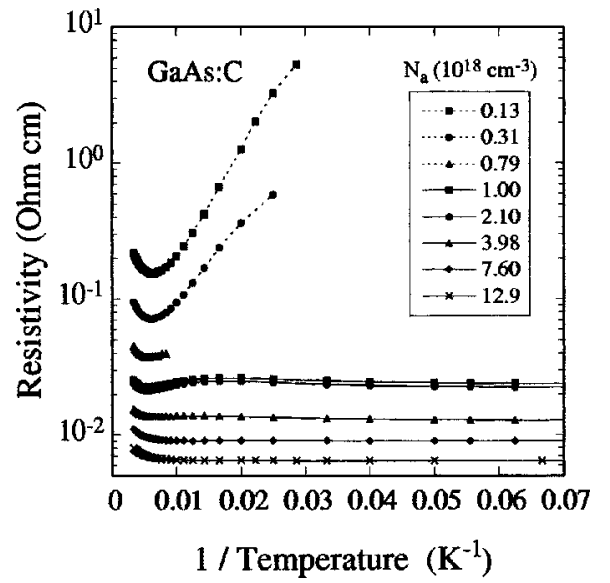

FIG. 1. Resistivity on a logarithmic scale versus inverse temperature for GaAs:C with different acceptor impurity concentrations.

and is compared to the result from a strict many-body calculation. The dynamic relaxation time is then identified and put back into Eq. (1). The net effect of the GDA is derived in the more general case of a polar semiconductor; the procedure is described in more detail in Ref. 14. The resistivity $\rho$ is then obtained as $1 / \sigma$ and is found to be

$$
\begin{aligned}
\rho(\omega)= & \frac{-i m^{*} \omega}{N e^{2}}-\frac{2 i}{3 \pi N \omega} \int_{0}^{\infty} q^{2} \\
& \times \frac{[\alpha(q, \omega)-\alpha(q, 0)]\left[\epsilon_{L}(q, \omega)+\alpha(q, 0)\right]}{\left[\epsilon_{T}^{2}(q, 0)\right]\left[\epsilon_{T}(q, \omega)\right]} d q .
\end{aligned}
$$

In the Eqs. (1) and (2), $N$ is the density of ionized acceptors. The acceptor impurity concentrations $N_{a}$ and $N$ are equal to each other on the metal side of the transition, but not on the insulating side. In Eqs. (1) and (2), $e$ is the electric charge, $m^{*}$ is the effective mass, $\alpha$ is the polarizability from the carriers, $\epsilon_{L}$ is the lattice dielectric function, $\epsilon_{T}$ is the total dielectric function, $\omega$ is the frequency variable, and $q$ is the momentum variable. ${ }^{8}$

The static resitivity in a polar semiconductor is the same as for a nonpolar semiconductor, for which the expressions are simpler. Since we are only interested in the static results, we used the expression for a nonpolar semiconductor as a starting point. According the GDA, the resistivity is reduced to $^{10}$

$$
\begin{aligned}
\rho(\omega)= & \frac{-i m^{*} \omega}{N e^{2}}+\frac{2 i}{3 \pi N \omega} \\
& \times \int_{0}^{\infty} q^{2}\left[\frac{1}{\epsilon_{T}(q, \omega)}-\frac{1}{\epsilon_{T}(q, 0)}\right] d q .
\end{aligned}
$$

We have assumed a random distribution of Coulomb impurities. The total dielectric function is given by ${ }^{8}$

$$
\epsilon_{T}(q, \omega)=\epsilon+\alpha_{1}(q, \omega)+i \alpha_{2}(q, \omega),
$$

where $\epsilon$ is the dielectric constant of GaAs and $\alpha_{1}$ and $\alpha_{2}$ are respectively, the real and imaginary parts of the polarizabilities of the dopant carriers. These functions are temperature dependent, and $\epsilon=12.4$ in GaAs. The imaginary part can be obtained analytically in the random phase approximation. It is given by

$$
\begin{aligned}
& \alpha_{2}(Q, \Omega)=\frac{-m^{*} e^{2}}{8 \hbar k_{\mathrm{F}} Q^{3} B} x \\
& \quad\left[\ln \left(\frac{\cosh \left\{B\left[\Omega+\left(Q^{2}+\Omega^{2} / Q^{2}-M\right) / 2\right]\right\}}{\cosh \left\{B\left[\Omega-\left(Q^{2}+\Omega^{2} / Q^{2}-M\right) / 2\right]\right\}}\right)-2 B \Omega\right],
\end{aligned}
$$

where we have introduced the dimensionless variables $Q$ $=q / 2 k_{\mathrm{F}}, \Omega=\hbar \omega / 4 E_{\mathrm{F}}, B=\beta E_{\mathrm{F}}$ and $M=\mu / E_{\mathrm{F}}$. The quantity $k_{\mathrm{F}}$ is the Fermi wave vector, given by $k_{\mathrm{F}}=\left(3 N \pi^{2}\right)^{1 / 3}$, and $E_{\mathrm{F}}$ is the Fermi energy. The real part can be obtained from the imaginary part through the Kramers-Kronig dispersion relation. We are interested in the static resistivity, which can be written as

$$
\rho(0)=\frac{4 \hbar k_{\mathrm{F}}^{3}}{3 \pi N E_{\mathrm{F}}} \int_{0}^{\infty} Q^{2} \frac{\left.\left[\partial \alpha_{2}(Q, \Omega) / \partial \Omega\right]\right|_{\Omega=0}}{\left[\epsilon+\alpha_{1}(Q, 0)\right]} d Q .
$$

This can be reduced to

$$
\rho(0)=\frac{2\left(m^{*} e\right)^{2}}{3 \pi N \hbar^{3} k_{\mathrm{F}}} \int_{0}^{\infty} \frac{\left\{1-\tanh \left[0.5 B\left(Q^{2}-M\right)\right]\right\}}{Q\left[\epsilon+\alpha_{1}(Q, 0)\right]} d Q .
$$

The chemical potential $\mu$ is obtained from the implicit expression $^{8}$

$$
B^{3 / 2}=\int_{0}^{U} \frac{3 y}{1-y^{2}} d Q\left\{A+\ln \left[\left(1-y^{2}\right) / y^{2}\right]\right\},
$$

where $U=\left(1+e^{-A}\right)^{-1 / 2}$ and $A=B M=\mu \beta$. For a given $A$, one obtains $B$, leading to a relation between them.

The calculated resistivity of GaAs:C as a function of impurity concentration and temperature, obtained using the procedure just described, is compared to the measured resistivity in the next section.

\section{RESULTS AND DISCUSSION}

Figures 1 and 2 show, respectively, the measured and calculated $\rho$ as a function of $1 / T$ for different values of $N_{a}$. The samples with $N_{a}$ higher than $1.0 \times 10^{18} \mathrm{~cm}^{-3}$ shown at the bottom of both figures display metallic behavior. These figures show the slope of the resistivity curves decreasing with increasing impurity concentration in the lowtemperature region down to our lowest temperature of $1.7 \mathrm{~K}$.

The most apparent difference between the experimental and theoretical results is the upturn of all the experimental curves in Fig. 1 at the high-temperature end. This upturn, which is absent in Fig. 2, is due to scattering from phonons, which becomes dominant above $\sim 100 \mathrm{~K}$. The phonon scattering was not taken into account in the theoretical calculations and consequently the high-temperature upturn is not reproduced in the theoretical curves of Fig. 2. 


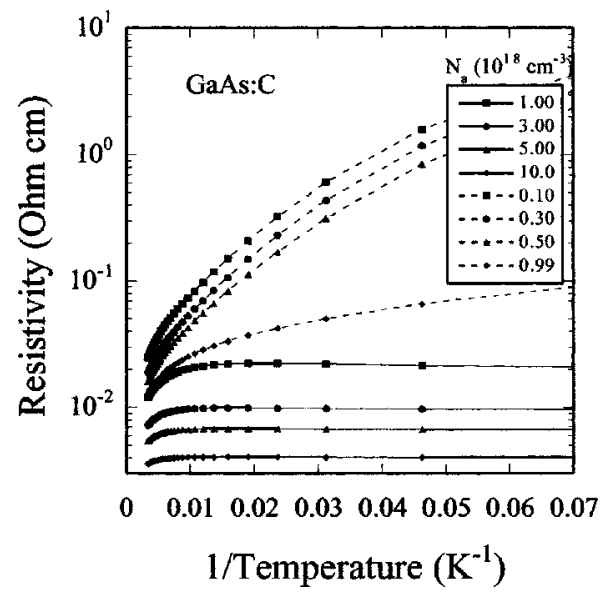

FIG. 2. Calculated resistivity of GaAs:C with different acceptor impurity concentration versus the inverse temperature.

For samples with $\mathrm{C}$ acceptor concentration less than $1.0 \times 10^{18} \mathrm{~cm}^{-3}$, the large enhancement in the resistivity as the temperature decreases, due to the reduction of free electrons in the conduction band, demonstrates the semiconductor properties of the GaAs:C samples in this range of $N_{a}$. The conduction-band activation energy $E_{a}$ is calculated from the slope of the $\rho$ versus $(1 / T)$ curves in Fig. 1 . The value $E_{a}=26 \mathrm{meV}$, considered as the acceptor ionization energy of $\mathrm{C}$ in GaAs, was found for the most diluted sample with $N_{a}$ $=1.3 \times 10^{17} \mathrm{~cm}^{-3}$, in agreement with the value obtained by photoluminescence measurements. ${ }^{15}$

Figures 3 and 4 show, respectively, measured and calculated results for the dependence of the resistivity of GaAs:C on the acceptor impurity concentration $N_{a}$ at different temperatures. One notices that all curves merge together to one critical point at about $1.0 \times 10^{18} \mathrm{~cm}^{-3}$, which is determined to be the critical concentration $N_{c}$ for the MNM transition in $p$-type GaAs:C. For impurity concentration above this point (i.e., the metallic region) the temperature dependence of the resistivity is negligible in comparison with the nonmetallic region.

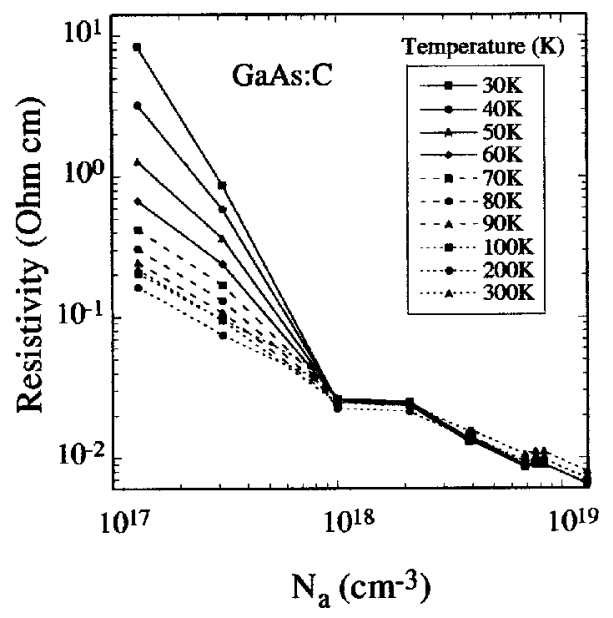

FIG. 3. Log- $\log$ plot of the resistivity of GaAs:C samples as a function of the acceptor impurity concentration at different temperatures.

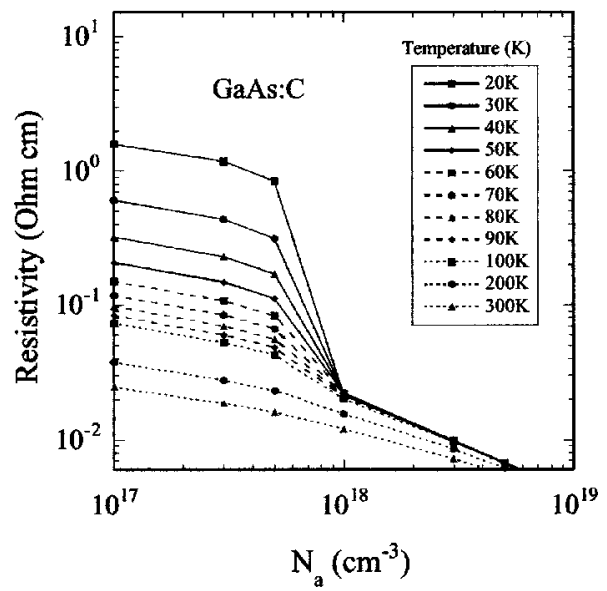

FIG. 4. On a double logarithmic scale, calculated resistivity of GaAs:C as a function of the acceptor impurity concentration at different temperatures.

To determine the value of $N_{c}$, we may also use three different computational methods as in Ref. 12; that is, the original Mott model, an extended Mott-Hubbard model, and a method based on total energy of the metallic and the nonmetallic phases. In the total energy model, the critical concentrations for the MNM transition are obtained from the total energy of the hole gas in the very nonparabolic valenceband curvature (including both heavy and light-hole bands). In the Mott picture, however, the critical concentration is obtained from the effective mass approximation, not considering scattering between the two uppermost valence bands. Therefore, one expect some differences for especially $p$-type material since the valence-band maximum is both degenerate and very nonparabolic. However, all three methods give rather similar values for the critical concentration. Here, we only present the results. Using these three methods, we obtained values for $N_{c}$ of $1.4 \times 10^{18}, 1.14 \times 10^{18}$, and 4.6 $\times 10^{17} \mathrm{~cm}^{-3}$, respectively. It is worth noting that the value of $N_{c}$ is about the same for $p$-type GaAs:C and $n$-type GaN:Si for both wurtzite and zinc-blende structures. ${ }^{12}$ In a simple picture, $N_{c} \sim\left(0.25 / a_{\mathrm{B}}\right)^{3}$, where the Bohr radius $a_{\mathrm{B}}$ is directly related to the ionization energy. Therefore, the similar ionization energies of GaAs and GaN give rather similar $N_{c} .{ }^{12}$

\section{SUMMARY}

In summary, the resistivity of GaAs implanted with carbon acceptors for concentrations spanning the insulating to the metallic regimes were investigated experimentally and theoretically between room temperature and $1.7 \mathrm{~K}$. Good agreement was obtained between the experimental results and calculations using a generalized Drude approach. The value of the critical impurity concentration $N_{c}$ for the MNM transition was estimated from both methods discussed, as well as through three different computational methods.

\section{ACKNOWLEDGMENTS}

We are indebted to Professor Myriam P. Sarachik for valuable discussions and help during the course of this project. This work was financially supported in part by the Brazilian National Research Council (CNPq), CNPq/ 
NanoSemiMat under grant no. 550.015/01-9, the Swedish Foundation for International Cooperation in Research and Higher Education (STINT), Swedish Research Council (VR), ATOMICS, and by the US Department of Energy Grant No. DE-FG02-84ER4515.

${ }^{1}$ S. Adachi, GaAs and Related Materials: Bulk Semiconducting and Superlattice Properties (World Scientific, Singapore, 1994).

${ }^{2}$ L. Pavesi and M. Guzzi, J. Appl. Phys. 75, 4779 (1994).

${ }^{3}$ I. Vurgaftman, J. R. Meyer, and L. R. Ram-Mohan, J. Appl. Phys. 89, 5815 (2001).

${ }^{4}$ J. Shirakashi, T. Azuma, F. Fukuchi, M. Konagai, and K. Takahashi, J. Cryst. Growth 150, 585 (1995).

${ }^{5}$ J. C. Zolper, M. E. Sherwin, A. G. Baca, R. J. Shul, J. F. Klem, and V. M. Hietala, IEEE Electron Device Lett. 15, 493 (1994).
${ }^{6}$ I. Danilov, J. P. de Souza, A. V. Murel, and M. A. A. Pudenzi, Appl. Phys. Lett. 78, 1700 (2001).

${ }^{7}$ L. J. Van der Pauw, Philips Res. Rep. 13, 1 (1958).

${ }^{8}$ B. E. Sernelius, Phys. Rev. B 36, 1080 (1987).

${ }^{9}$ B. E. Sernelius, Phys. Rev. B 41, 3060 (1990).

${ }^{10}$ A. Ferreira da Silva, B. E. Sernelius, J. P. de Souza, H. Boudinov, H. Zheng, and M. P. Sarachik, Phys. Rev. B 60, 15824 (1999).

${ }^{11}$ A. Ferreira da Silva, C. Moysés Araújo, B. E. Sernelius, C. Persson, R. Ahuja, and B. Johansson, J. Phys.: Condens. Matter 13, 8891 (2001).

${ }^{12}$ A. Ferreira da Silva and C. Persson, J. Appl. Phys. 92, 2550 (2002).

${ }^{13}$ J. F. Ziegler, J. P. Biersak, and U. Littmark, in The Stopping and Range of Ions in Solids (Pergamon, New York, 1985).

${ }^{14}$ B. E. Sernelius, Phys. Rev. B 40, 12438 (1989).

${ }^{15}$ Semiconductors-Basic Data, edited by O. Madelung, 2nd revised ed. (Springer, New York, 1996). 